\title{
Small Displacement Detection of Biological Signals Using the Cyclic Frequency Method
}

\author{
Dan Zhang, ${ }^{1,2}$ Masahiko Kurata, ${ }^{3}$ and Takayuki Inaba ${ }^{3}$ \\ ${ }^{1}$ College of Information Science and Technology, Nanjing Forestry University, Nanjing 210037, China \\ ${ }^{2}$ School of Systems, Kochi University of Technology, Kami, Kochi 782-8502, Japan \\ ${ }^{3}$ Graduate School of Informatics and Engineering, University of Electro-Communications, 1-5-1 Chofugaoka, Chofu, \\ Tokyo 182-8585, Japan
}

Correspondence should be addressed to Dan Zhang; zhangdan1227@hotmail.com

Received 1 October 2014; Revised 22 January 2015; Accepted 5 March 2015

Academic Editor: N. Nasimuddin

Copyright (C) 2015 Dan Zhang et al. This is an open access article distributed under the Creative Commons Attribution License, which permits unrestricted use, distribution, and reproduction in any medium, provided the original work is properly cited.

A new signal processing method called the Cyclic Frequency method is proposed for small displacement detection of vital signals such as heart rate and respiration using the CW radar method. We have presented experimental results of small displacement detection to confirm the validity of the method. The displacement amplitude $2.5 \mathrm{~mm}$ can be detected with a propagation frequency of $24.15 \mathrm{GHz}$. We may increase the propagation frequency for smaller displacement amplitude or target velocity.

\section{Introduction}

Microwave Doppler radar with quadrature direct conversion is a promising method for noncontact detection and monitoring of human cardiopulmonary activities. Because Doppler radar can detect respiration and heartbeat rates in a noninvasive way, some patients no longer experience pain, especially burn victims, and disruptions to the activity of subjects can be minimised. Thus, its application is more suitable when extended monitoring is required, such as health monitoring. In addition to its potential uses in health care, Doppler radar can be applied in emergency rescue situations such as earthquakes and fire rescue, where it is impossible to reach subjects trapped under debris. It can also be used to quickly locate victims. Furthermore, due to its ability to penetrate walls, Doppler radar can be used for security in places where noncooperative subjects are present, such as airports and military checkpoints.

CW (continuous wave) radar has the advantages of low power consumption and simple radio architecture. However, it is usually difficult to distinguish heart rate signals from components of respiratory fluctuation. Therefore, some signal processing and radar technologies, such as multipleinput and multiple-output techniques for the detection of multiple subjects $[1,2]$, robust spectral RELAX algorithm for accurately discriminating heartbeat and respiration frequencies [3], adaptive noise cancellation for extracting heart rate signals [4], and the MEM method [5] based on Fourier transform, have been proposed.

Heart rate signals are concealed in respiratory signals. Because of this, we proposed a projection matrix method in previous work to suppress unnecessary and periodic components of fluctuation and reduce the effects of harmonics [6], which is based on the correlation matrix principle. Usually, body surface displacement velocities due to respiration and the heart are very different, and they can be distinguished by different Doppler frequencies. However, if displacement reaches almost the same velocity then it is difficult to read respiratory signals and heart rate signals using this method. In this paper, we aim to introduce a new signal processing method in order to clearly separate them. The short time Fourier transform is used to obtain the Doppler frequency which is shown along the vertical axis. Fourier transform is then executed to obtain the Cyclic Frequency, which is shown along the horizontal axis. The same approach was used to investigate the recognition of the walking motion of humans in previous literature [7]. The results show that it is also 


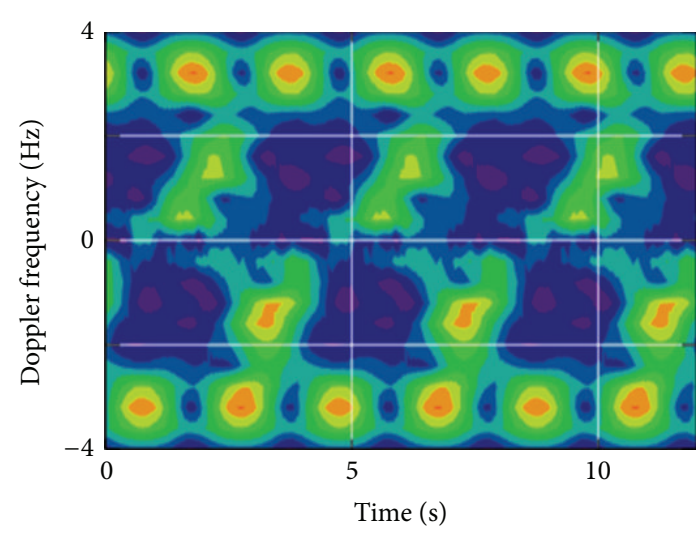

(a) Spectrogram

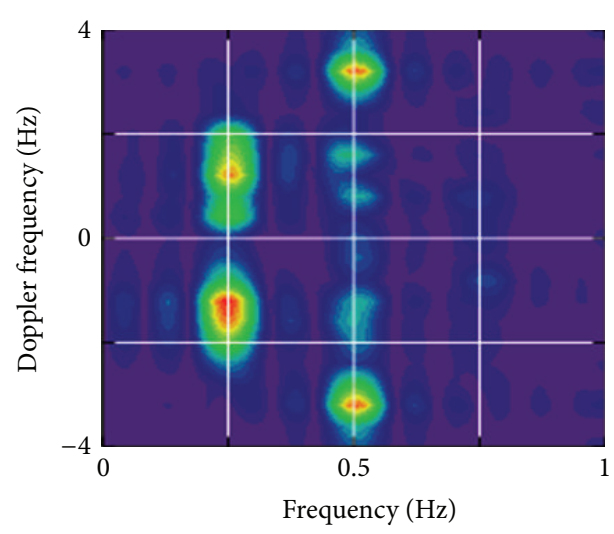

(b) Cyclic Frequency

FIGURE 1: Cyclic Frequency method, the proposed data processing method.

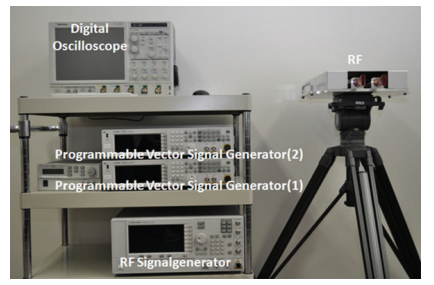

(a) Photo of the radar system setup

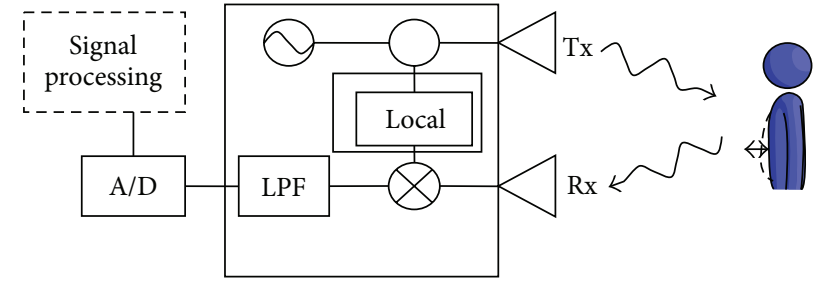

(b) Block diagram of the radar system

FIGURE 2: The proposed experimental radar system.

possible to separate respiratory signals and heart rate signals even with the same displacement velocity.

\section{Materials and Methods}

2.1. Cyclic Frequency Method. Sending a constant CW wave and mixing the received wave with the transmitted wave through the LPF (low pass filter), we obtain the following beat signal [8]:

$$
\begin{aligned}
& B(t)=A e^{j\left(2 \pi f_{d} t+\varphi\right)} \text { with } \\
& f_{d}=\frac{2 v}{\lambda},
\end{aligned}
$$

where $v$ is the target velocity (receding direction is positive), $\lambda$ is the wavelength of the transmitted wave, and $\varphi$ is the phase value determined by the initial target distance. During the observation period, Fourier transform is performed for the beat signal and the frequency spectrum becomes the Doppler frequency $f_{d}=2 v / \lambda$ corresponding to velocity. If the target is displaced periodically, then the same frequency spectrum will appear during each displacement period. Therefore, by determining the frequency of the temporal variation of the Doppler frequency spectrum, called Cyclic Frequency, we can obtain the displacement period.

First, we perform Fourier transform on the beat signal during the predetermined observation period to obtain a Doppler frequency spectrum. Then, we adjust the observation period and repeat Fourier transform. This well-known process is STFT (short time Fourier transform). The frequency spectrum is arranged in the time direction as shown in Figure 1(a). The horizontal axis is time and the vertical axis is Doppler frequency, while the figure is called a spectrogram.

Next, performing Fourier transform in the time direction for each Doppler frequency in the spectrogram, we can obtain Figure 1(b). The period of displacement can be determined from the peak frequency position in the figure. Because the Doppler frequency and period of displacement of heart rate signals are very different compared with those of respiratory signals, this method distinguishes between heart rate signals and respiratory signals quite well even if they have the same displacement velocity. We call this method the Cyclic Frequency method.

\section{Results and Discussion}

The experiment was performed using the radar software developed in our laboratory, as shown in Figure 2. The target was the human body. The radar software was placed 1.8 metres in front of the target. The propagation frequency was $24.15 \mathrm{GHz}$, the sampling frequency was $10 \mathrm{kHz}$, the Fourier transform period was $2.5 \mathrm{~s}$, and the adjustment time was $0.1 \mathrm{~s}$. For the simulated experiment, we assumed a target constant velocity of $5 \mathrm{~mm} / \mathrm{s}$ and the target displacement period and amplitude were set at $4 \mathrm{~s}$ and $10 \mathrm{~mm}$, respectively. As shown in Figure 3, the figure on the left is called a spectrogram and the figure on the right shows the results using the 


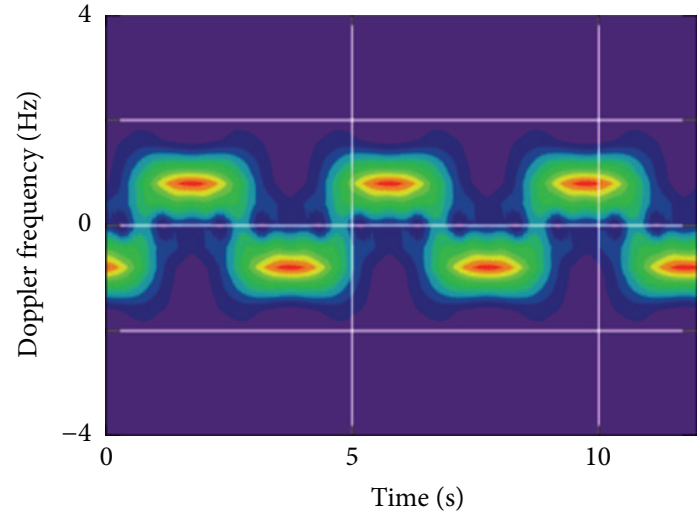

(a) Spectrogram

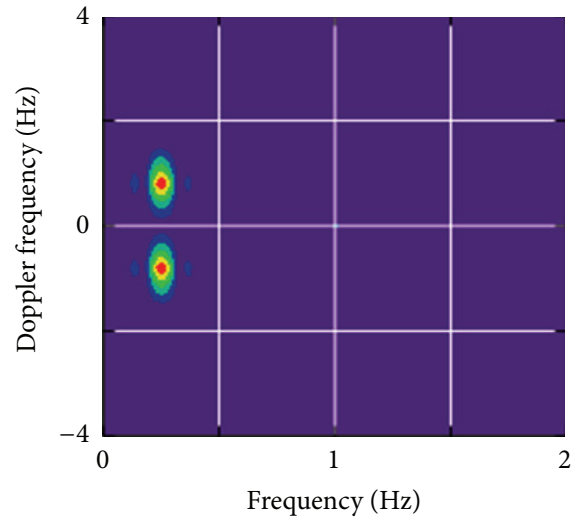

(b) Cyclic Frequency

FIgURE 3: The proposed data processing method with a displacement period of $1 \mathrm{~s}$.

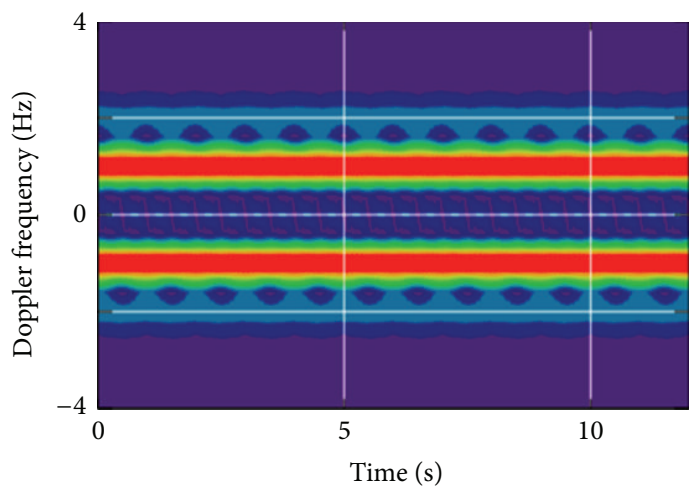

(a) Spectrogram

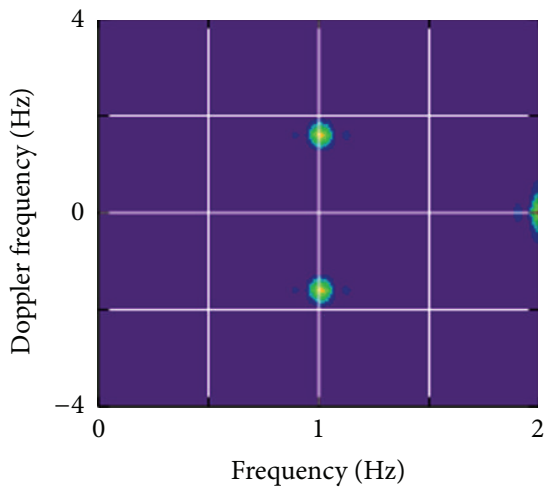

(b) Cyclic Frequency

FIGURE 4: The simulation results with a displacement period of $1 \mathrm{~s}$.

Cyclic Frequency method. The Cyclic Frequency value was discovered to be $0.25 \mathrm{~Hz}$, exactly equivalent to the period of the target displacement.

In order to verify the volume of the displacement period and amplitude detected using the Cyclic Frequency method according to the above-mentioned conditions, we adjusted the displacement period to $1 \mathrm{~s}$ and $0.5 \mathrm{~s}$. This was equivalent to the amplitude being adjusted from $2.5 \mathrm{~mm}$ to $1.25 \mathrm{~mm}$. The results are shown in Figures 4 and 5. For the displacement period of $0.5 \mathrm{~s}$, it was impossible to obtain a clear peak in the Cyclic Frequency figure, and, in the spectrogram figure, it is hard to see that the Doppler frequency is $0.80 \mathrm{~Hz}$ (period of $1.25 \mathrm{~s})$.

Following this, we paid more attention to the details when the displacement period was adjusted to $0.5 \mathrm{~s}$ and $4 \mathrm{~s}$. We extracted the real part of the beat signal and the target displacement, which are shown in Figure 6. At $4 \mathrm{~s}$, the target displacement period was approximately three times the Doppler frequency period in the beat signal. At $0.5 \mathrm{~s}$, the target displacement period was about 0.4 times the Doppler frequency period. From this, we can see that the Doppler frequency periodic component was reduced during the target displacement period. This means that if the displacement period or velocity becomes smaller, the Doppler frequency periodic component will decrease during the period. In this condition, it is more difficult to use the Cyclic Frequency method.

In Figure 7, the curves show the relationship between displacement period and target velocity at certain propagation frequencies. On each curve, the Doppler frequency component is included for one period. As the propagation frequency increases, the zone of the displacement period and target velocity (upper zone of the curve) widens. This shows that we can improve the propagation frequency for smaller displacement periods or target velocities using the Cyclic Frequency method.

\section{Conclusions}

We proposed a method called the Cyclic Frequency method for detecting small displacement such as respiration and heartbeat. Our simulation shows that when propagation frequency is $24 \mathrm{GHz}$, we can measure displacement amplitude of $2.5 \mathrm{~mm}$, which corresponds to one second of the target displacement period. Furthermore, we can improve the 


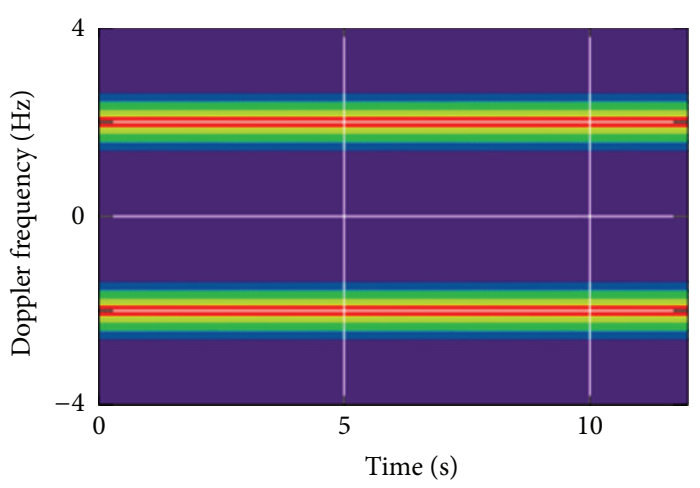

(a) Spectrogram

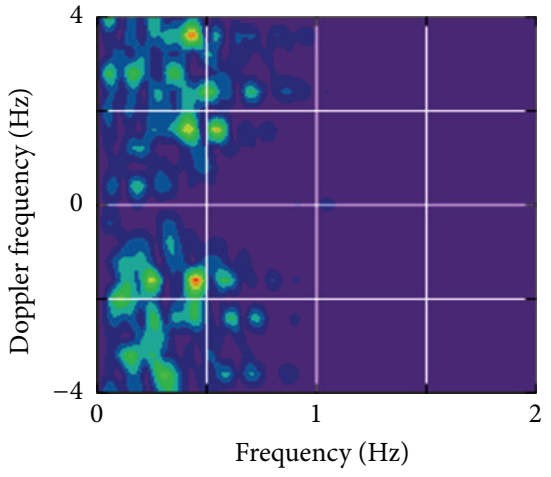

(b) Cyclic Frequency

FIgURE 5: The simulation results with a displacement period of $0.5 \mathrm{~s}$.

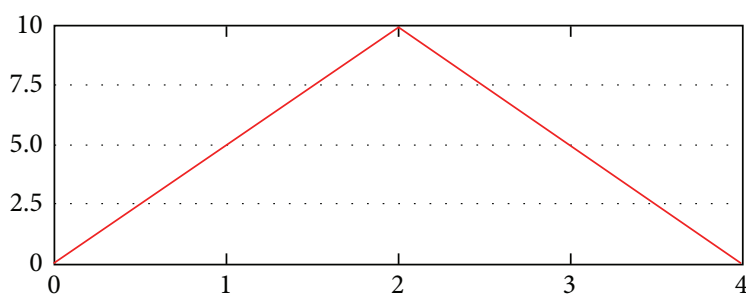

(1) Displacement signal

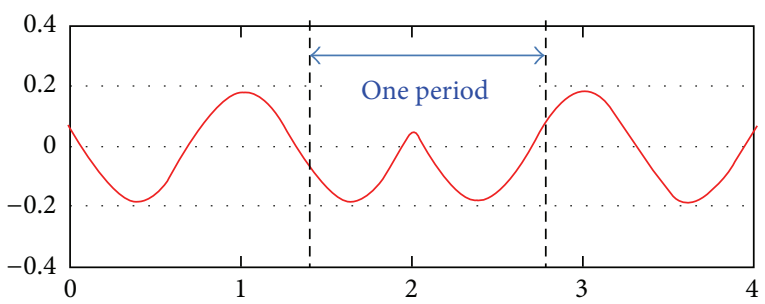

(2) Real part of the beat signal

(a) Target displacement period of $4 \mathrm{~s}$

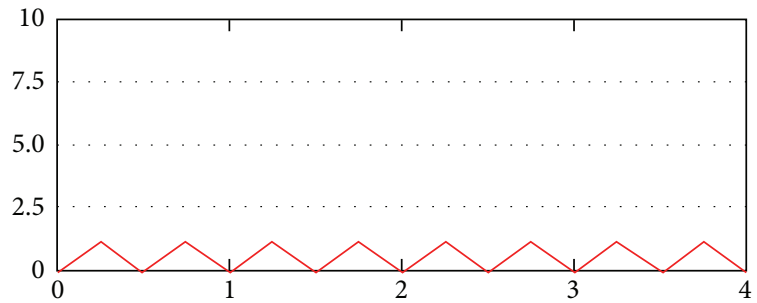

(1) Displacement signal

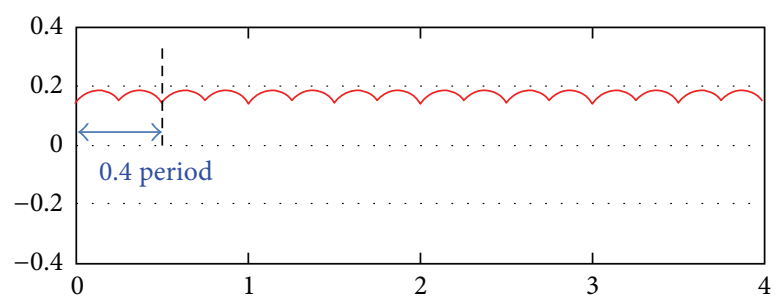

(2) Real part of the beat signal

(b) Target displacement period of $0.5 \mathrm{~s}$

FIGURE 6: The target displacement and real part of the beat signal.

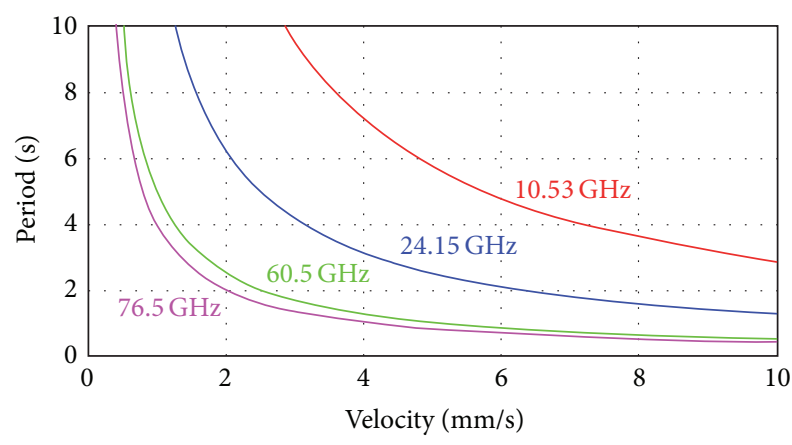

FIGURE 7: Relationship between displacement periods and target velocities for propagation frequency.

propagation frequency for smaller displacement periods or target velocities using the Cyclic Frequency method.

\section{Conflict of Interests}

The authors declare that there is no conflict of interests regarding the publication of this paper.

\section{References}

[1] O. Boric-Lubecke, V. M. Lubecke, A. Host-Madsen, D. Samardzija, and K. Cheung, "Doppler radar sensing of multiple subjects in single and multiple antenna systems," in Proceedings of the 7th International Conference on Telecommunications in Modern Satellite, Cable and Broadcasting Services (TELSIKS '05), vol. 1, pp. 7-11, IEEE, September 2005.

[2] Q. Zhou, J. Liu, A. Høst-Madsen, O. Boric-Lubecke, and V. Lubecke, "Detection of multiple heartbeats using Doppler radar," in Proceedings of the IEEE International Conference on Acoustics, Speech and Signal Processing (ICASSP'06), pp. II1160II1163, May 2006. 
[3] C. Li, J. Ling, J. Li, and J. Lin, "Accurate doppler radar noncontact vital sign detection using the RELAX algorithm," IEEE Transactions on Instrumentation and Measurement, vol. 59, no. 3, pp. 687-695, 2010.

[4] D. R. Morgan and M. G. Zierdt, "Novel signal processing techniques for Doppler radar cardiopulmonary sensing," Signal Processing, vol. 89, no. 1, pp. 45-66, 2009.

[5] D. Nagae and A. Mase, "Measurement of heart rate variability and stress evaluation by using microwave reflectometric vital signal sensing," Review of Scientific Instruments, vol. 81, no. 9, Article ID 094301, 2010.

[6] D. Zhang, M. Kurata, and T. Inaba, "FMCW radar for small displacement detection of vital signal using projection matrix method," International Journal of Antennas and Propagation, vol. 2013, Article ID 571986, 5 pages, 2013.

[7] C. Hornsteiner and J. Detlefsen, "Characterisation of human gait using a continuous-wave radar at $24 \mathrm{GHz}$," Advances in Radio Science, vol. 6, pp. 67-70, 2008.

[8] M. Kurata and T. Inaba, "A study of small displacement measurement using microwave radar," in Proceedings of the IEICE General Conference, pp. B-2-B-56, Gifu, Japan, March 2013. 

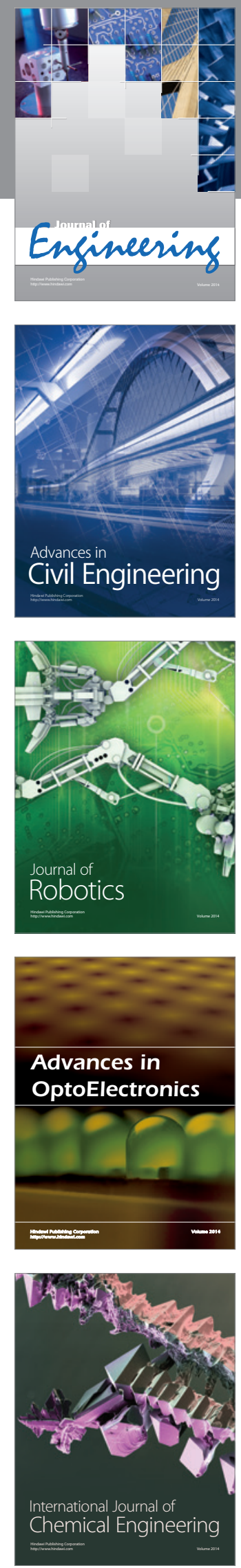

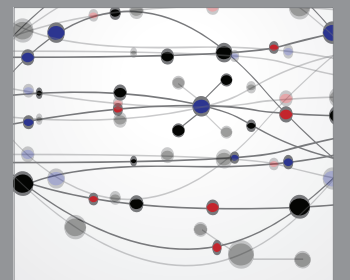

The Scientific World Journal
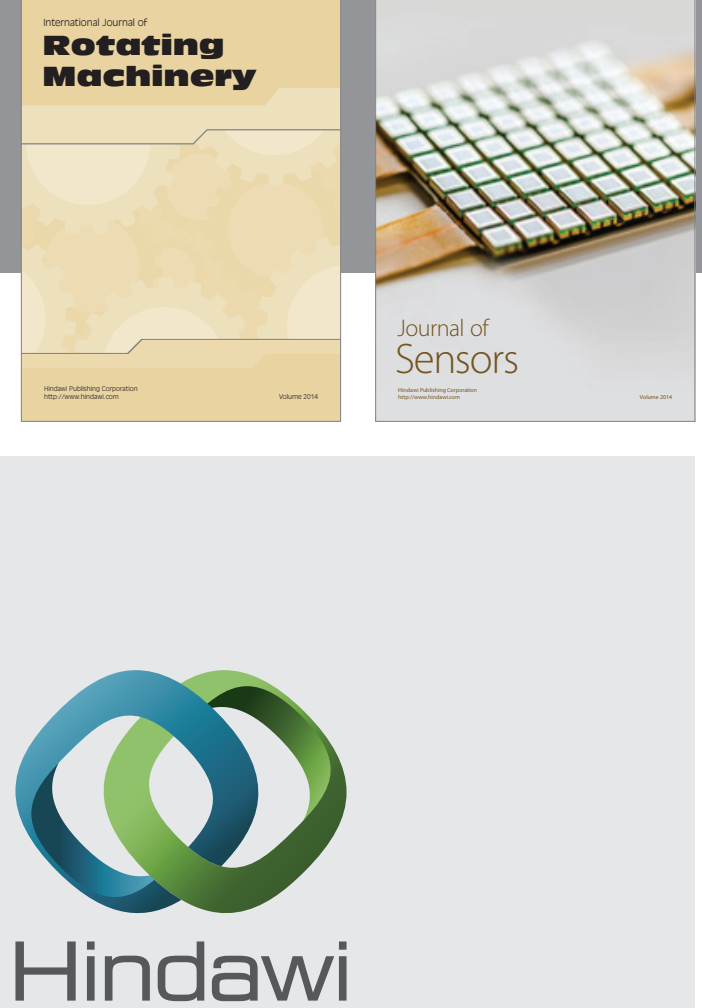

Submit your manuscripts at http://www.hindawi.com
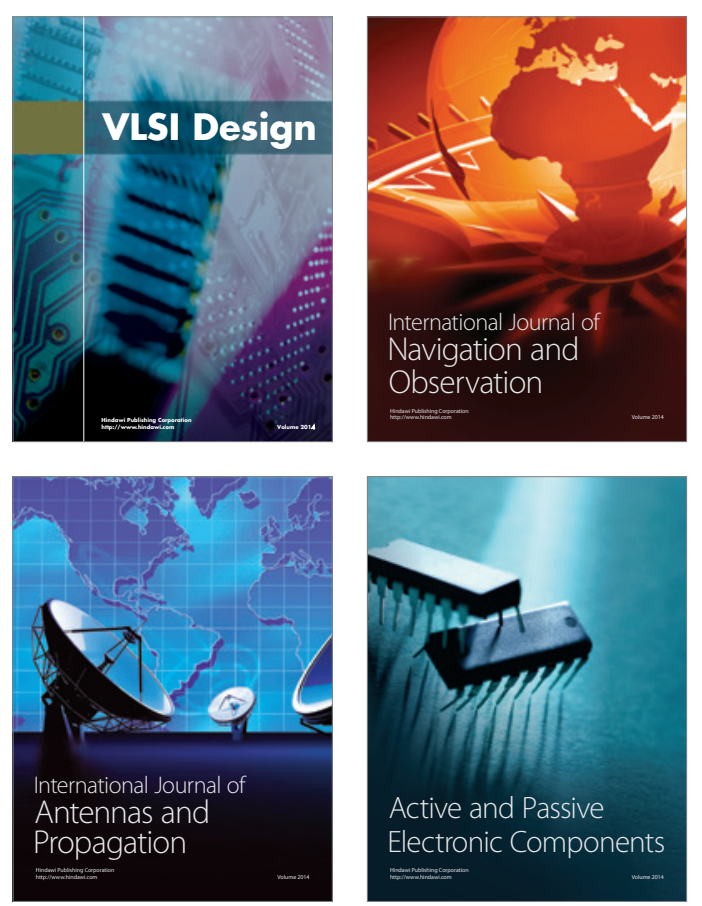
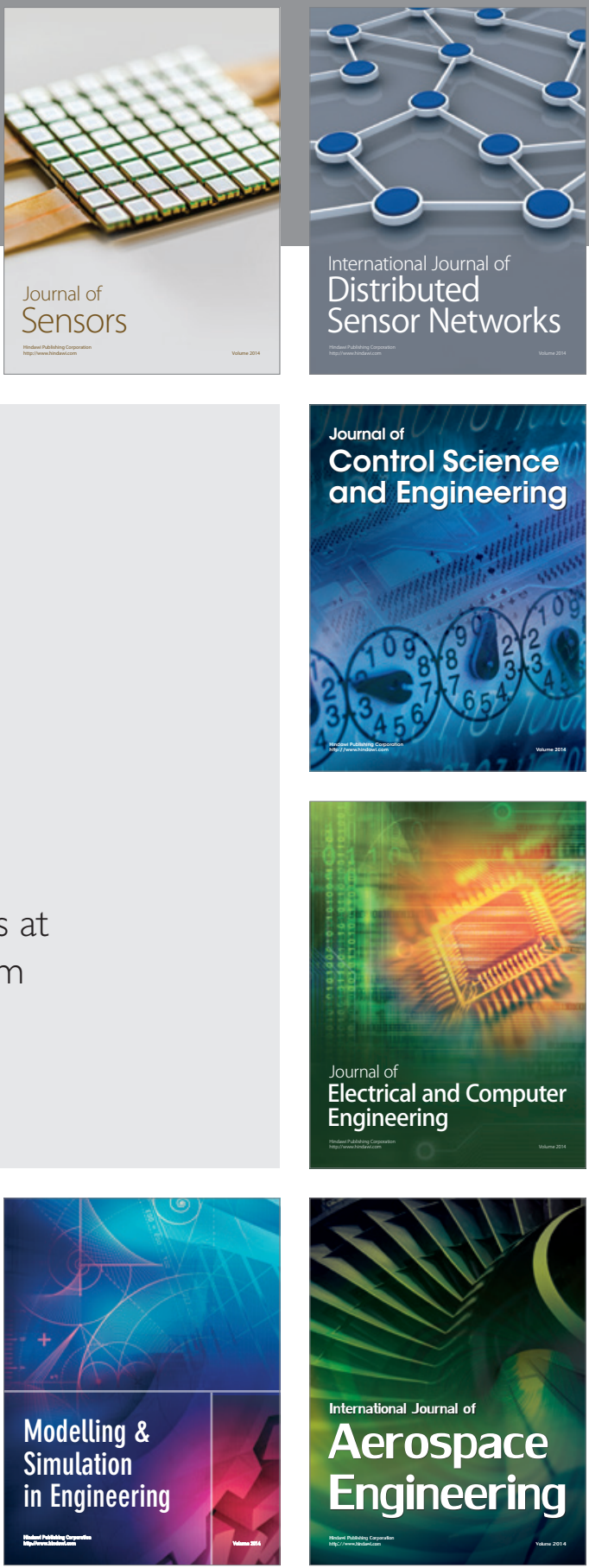

Journal of

Control Science

and Engineering
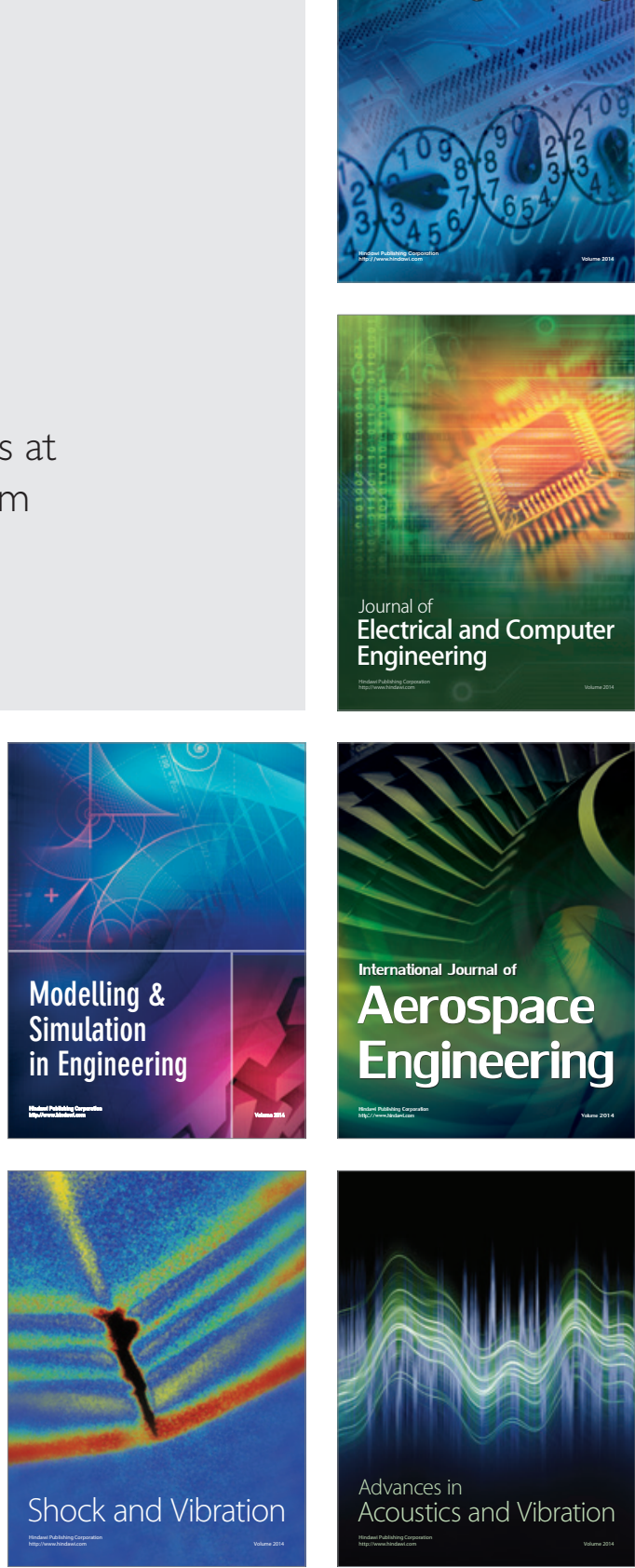